\title{
CHARACTERISTICS OF ALLERGIC REACTIONS TO DRUGS IN CHILDREN
}

\author{
V. Bulgakova1, T. Eliseeva², E. Tush², E. Zastelo², O. Khaletskaya², S. Ignatov', I. Balabolkin ${ }^{1}$ \\ ${ }^{1}$ National Medical Research Center of Children's Health, Institute of Pediatrics, Moscow, Russia. \\ ${ }^{2}$ Privolzhsky Research Medical University, Department of Pediatrics, Nizhny Novgorod, Russia. \\ ${ }^{3}$ Lobachevsky State University of Nizhny Novgorod, Department of Chemistry, Nizhny Novgorod, Russia.
}

\section{Background and Aims}

Drug allergy may produce a rash and other unwanted symptoms and signs.

\section{Method}

A retrospective analysis of medical records of 76 patients aged 3-17 years admitted to a children's hospital in 2015-2017 in connection with acute hypersensitivity reactions to drugs was carried out.

Clinical characteristics of The data are presented as absolute patients

Number of patients

Age value / group fraction (\%)

\section{1}

$7.52 \pm 0.95$

$[6.57 ; 8.47]$

Age (month)

Boys

Girls

Prescribed therapy:

Glucocorticosteroids

dose (mg/kg per day)

Clinical syndromes:

Urticaria

Urticaria + Quincke's edema

Quincke's edema

Other skin rashes

Other skin rashes +

Quincke's edema

Serum sickness

Anaphilaxis
$2.57 \pm 0.61$

$[1.95 ; 3.19]$

$95.02 \pm 11.53$

[83.49; 106.56]

33

38

23/32.39

$11 / 15.49$

$3 / 4.23$

$11 / 15.49$

$16 / 22.54$

$1 / 1.41$

$6 / 8.45$

\section{Results}

The average age of patients was 7.6 years [7.0-8.2]: 34 boys, 42 girls. In 5/76 (6.8\%) patients reported cases of anaphylaxis (3 on topical anesthetics at dental interventions, 1 on the administration of an antirabic immunoglobulin, 1 on intramuscular injection of cephalosporins). Angioedema developed in a 6-year-old girl after oral administration of NSAIDs for headache relief. Toxico-epidermal necrolysis was diagnosed in a 5year-old girl after using the multivitamins. In $69 / 76$ (90.8\%) patients there were various rashes - urticaria, spotted-papular and morbilliform exanthema. Thus, in most children, cutaneous manifestations of hypersensitivity prevailed. Hypersensitive reactions to drugs of the type of acute urticaria are diagnosed in $21 / 76(27.6 \%)$. In $17 / 76$ (22.4\%) children there was a combined development of urticaria rash and angioedema. Thus, all clinical manifestations of acute urticaria occurred in 38/76 (50\%) patients. Skin rash variants, including spotted-papular and morbilliform exanthema, which were quite resistant to therapy, in some cases prone to pigmentation, occurred in 31/76 (40.8\%) patients, while 19 of them marked by a combination of such rashes and symptoms Quincke's oedema. In total, angioedema combined with cutaneous manifestations of hypersensitivity occurred in 36/76 (47.4\%) patients hospitalized for drug hypersensitivity reactions.

\section{Conclusion}

The most common clinical manifestations of side effects on medicines in children are urticaria and morbilliform exanthema.
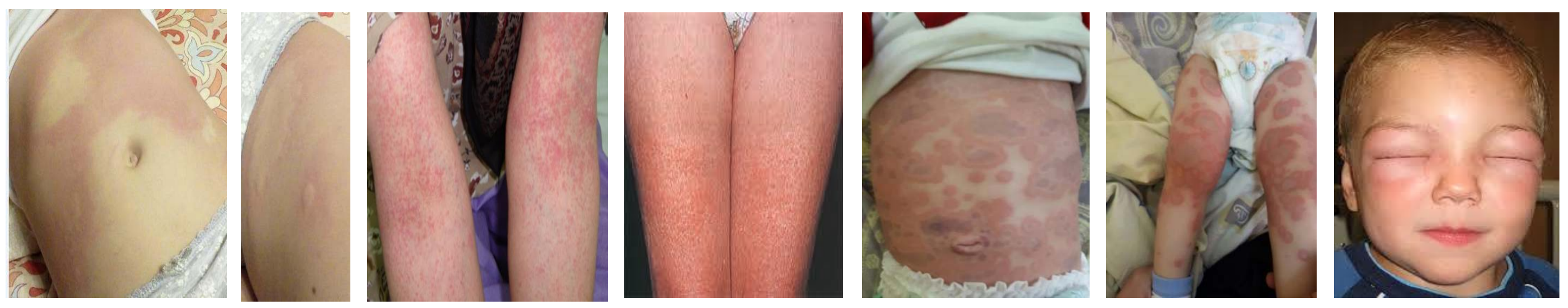

\section{References}

Demoly P., Adkinson N.F., Brockow K., Castells M., Chiriac A.M., Greenberger P.A., Khan D.A., Lang D.M., Park H.S., Pichler W., SanchezBorges M., Shiohara T., Thong B.Y. International Consensus on drug allergy. Allergy 2014; 69(4): 420-437.

Castells MC. Drug Allergy: Phenotypes, Endotypes, and Biomarkers. J Allergy Clin Immunol Pract. 2017 May - Jun;5(3):626-627. 